\title{
COHOMOLOGY THEORY FOR NON-NORMAL SUBGROUPS AND NON-NORMAL FIELDS*
}

\author{
by IAIN T. ADAMSON \\ (Received 20th September, 1953)
}

1. Introduction. Let $G$ be a finite group, $H$ an arbitrary subgroup (i.e., not necessarily normal) ; we decompose $G$ as a union of left cosets modulo $H$ :

$$
G=\mathrm{U}_{\nu} \bar{x}_{\nu} H
$$

choosing fixed coset representatives $\bar{x}_{\nu}$. In this paper we construct a " coset space complex" and assign cohomology groups; $H^{r}([G: H], A)$, to it for all coefficient modules $A$ and all dimensions, $-\infty<r<\infty$. We show that if

$$
0 \rightarrow A^{\prime} \rightarrow A \rightarrow A^{\prime \prime} \rightarrow 0
$$

is an exact sequence of coefficient modules such that $H^{1}\left(U, A^{\prime}\right)=0$ for all subgroups $U$ of $H$, then a cohomology group sequence

$$
\rightarrow H^{r}\left([G: H], A^{\prime}\right) \rightarrow H^{r}([G: H], A) \rightarrow H^{r}\left([G: H], A^{\prime \prime}\right) \rightarrow H^{r+1}\left([G: H], A^{\prime}\right) \rightarrow
$$

may be defined and is exact for $-\infty<r<\infty$. We also provide a link between the cohomology groups $H^{r}([G: H], A)$ and the cohomology groups of $G$ and $H$; namely, we prove that if $H^{\nu}(U, A)=0$ for all subgroups $U$ of $H$ and for $\nu=1,2, \ldots, n-1$, then the sequence

$$
0 \rightarrow H^{n}([G: H], A) \rightarrow H^{n}(G, A) \rightarrow H^{n}(H, A)
$$

is exact, where the homomorphisms of the sequence are those induced by injection, inflation and restriction respectively.

These results are then applied in defining cohomology groups for the coefficient groups which are associated in class field theory with an arbitrary field extension (i.e., the groups of idèles, idèle classes and the multiplicative group of non-zero elements of the field). These groups are defined by introducing an auxiliary normal extension; but are independent of the normal extension used. In the case of normal extensions, the groups here defined coincide with those used by Hochschild and Nakayama (5), and by Artin and Tate (1, 2). If $A$ is either the multiplicative group of non-zero elements of a local field $E$, or the group of idèle classes of a global field $E$, we shall show that $H^{1}(A)=0$, and that $H^{2}(A)$ is cyclic of order equal to the degree of $E$ over the ground field, and is generated by a canonical cohomology class. We shall also discuss an attempt to generalise to the non-normal case Tate's theorem (6) linking the cohomology groups of $A$ with those of the integers.

I wish to thank Professor Artin for his help and encouragement during the preparation of this paper, Dr. J. T. Tate for a number of fruitful discussions, and Mr. R. J. Semple for checking the laborious cocycle computations in an earlier proof of Theorem 7.3.

2. G-complexes. Let $G$ be a group, $\bar{G}$ its group ring with integer coefficients. A $G$-complex $K$ is a collection of left $\bar{G}$-modules, $C_{r}(K)(-\infty<r<\infty)$, called the chain groups of $K$,

* This paper is based on a dissertation presented to the Faculty of Princeton University in partial fulfilment of the requirements for the degree of Doctor of Philosophy, June 1952. 
and a collection of $\bar{G}$-homomorphisms, $\partial_{r}(-\infty<r<\infty)$, called the boundary operators of $K$,

$$
\partial_{r}: C_{r}(K) \rightarrow C_{r-1}(K) \text {, }
$$

such that $\partial_{r} \partial_{r+1}$ is the zero homomorphism.

For $-\infty<r<\infty$ we define the group of $r$-cycles, $Z_{r}(K)$, to be the kernel in $C_{r}(K)$ under $\partial_{r}$; the group of $r$-boundaries, $B_{r}(K)$, to be the image of $C_{r+1}(K)$ under $\partial_{r+1}$; and the $r$-dimensional homology group, $H_{r}(K)=Z_{r}(K) / B_{r}(K)$. A $G$-complex $K$ is said to be acyclic if all the homology groups $H_{r}(K)=0,(-\infty<r<\infty)$.

Now let $A$ be a left $G$-module. Then an $r$-cochain of $K$ with coefficients in $A$ is a $\bar{G}$-homomorphism of $C_{r}(K)$ into $A$. These cochains form an additive group, $C^{r}(K, A)$, under the addition defined by setting

$$
(f+g)(c)=f(c)+g(c), \quad f, g \in C^{r}(K, A), c \in C_{r}(K) .
$$

Coboundary operators, $\delta_{r}(-\infty<r<\infty)$,

$$
\delta_{r}: C^{r}(K, A) \rightarrow C^{r+1}(K, A)
$$

are defined by setting

$$
\left(\delta_{r} f\right)(c)=f\left(\partial_{r+1} c\right), \quad f \epsilon C^{r}(K, A), c \in C_{r+1}(K) .
$$

These coboundary operators are clearly homomorphisms and satisfy the conditions $\delta_{r} \delta_{r-1}=0$. We define the group of $r$-cocycles, $Z^{r}(K, A)$, to be the kernel in $C^{r}(K, A)$ under $\delta_{r}$; the group of $r$-coboundaries, $B^{r}(K, A)$, to be the image of $C^{r-1}(K, A)$ under $\delta_{r-1}$; and the $r$-dimensional cohomology group, $H^{r}(K, A)=Z^{r}(K, A) / B^{r}(K, A)$.

Let $A$ and $B$ be left $\bar{G}$-modules, $\theta$ a $\bar{G}$-homomorphism of $A$ into $B$. Then the homomorphisms

$$
\theta^{r}: C^{r}(K, A) \rightarrow C^{r}(K, B)
$$

defined by

$$
\left(\theta^{r} f\right)(c)=\theta(f(c)), \quad f \in C^{r}(K, A), c \in C_{r}(K),
$$

clearly satisfy the condition $\theta^{r} \delta_{r-1}=\delta_{r-1} \theta^{r-1}$, and hence induce homomorphisms of the corresponding cohomology groups,

$$
\theta_{*}^{r}: H^{r}(K, A) \rightarrow H^{r}(K, B) .
$$

3. Coset Space Complexes. Let $G$ be a group, $H$ an arbitrary subgroup of finite index in $G$. We denote the left cosets $x H$ of $G$ modulo $H$ by $A_{1}, A_{2}, \ldots$; if $A_{v}$ is one of these cosets, $x$ an element of $G$, then $x A_{v}$ is also one of the cosets.

We now construct a $G$-complex $K=K([G: H])$. For $r \geqslant 0$ the chain groups $C_{r}(K)$ are the free abelian groups generated by the $(r+1)$-tuples of cosets, $\left(A_{0}, \ldots, A_{r}\right)$; for $r \geqslant 1$, the chain groups $C_{-r}(K)$ are the free abelian groups generated by the $r$-tuples of cosets, $\left(A_{1}, \ldots, A_{r}\right)$ These chain groups become left $\bar{G}$-modules under the natural definition,

$$
x\left(\ldots, A_{v}, \ldots\right)=\left(\ldots, x A_{v}, \ldots\right), \quad x \in G .
$$

The boundary operators, $\partial_{r}$, are defined by setting

$$
\begin{aligned}
& \partial_{r}\left(A_{0}, \ldots, A_{r}\right)=\Sigma_{v=0}^{r}(-1)^{\nu}\left(A_{0}, \ldots, \hat{A}_{v}, \ldots, A_{r}\right), \quad r \geqslant 1 ; \\
& \partial_{0}\left(A_{0}\right)=\Sigma_{\Delta}(A) ; \\
& \partial_{-r}\left(A_{1}, \ldots, A_{r}\right)=\Sigma_{\Delta}\left(A, A_{1}, \ldots, A_{r}\right)+\Sigma_{\nu=1}(-1)^{\nu} \Sigma_{\Delta}\left(A_{1}, \ldots, A_{v}, A, A_{v+1}, \ldots, A_{r}\right), r \geqslant 1,
\end{aligned}
$$


and extending by linearity. (The circumflex denotes the omission of the argument over which it stands.) It is easily verified that these are $\bar{G}$-homomorphisms satisfying the condition $\partial_{r} \partial_{r+1}=0$. If $A$ is a left $\bar{G}$-module the cochains, ..., cohomology groups of $K([G: H])$, with coefficients in $A$ are called simply the cochains, ..., cohomology groups of the coset space $[G: H]$, and are denoted by $C^{r}([G: H], A), \ldots, H^{r}([G: H], A)$.

We remark that, if $G$ is a finite group, then the coset space complex $K([G: 1])$ obtained by taking $H=1$ is identical with the group complex $K_{G}$ of $G$ as defined (for positive dimensions) by Eilenberg (4) and extended to negative dimensions by Tate (2).

Theorem 3.1. The coset space complex $K([G: H])$ is acyclic.

Proof. We define mappings $\psi_{r}: C_{r}(K) \rightarrow C_{r+1}(K)$ by setting

$$
\begin{gathered}
\psi_{r}\left(A_{0}, \ldots, A_{r}\right)=\left(H, A_{0}, \ldots, A_{r}\right), r \geqslant 0 ; \\
\psi_{-1}(A)= \begin{cases}(H) & \text { if } A=H, \\
0 & \text { otherwise }\end{cases} \\
\psi_{-r}\left(A_{1}, \ldots, A_{r}\right)=\left\{\begin{array}{ll}
\left(A_{2}, \ldots,\right. & \left.A_{r}\right) \text { if } A_{1}=H, \\
0 & \text { otherwise, }
\end{array} \quad r \geqslant 2 .\right.
\end{gathered}
$$

Then it is easily verified that for any $r$-chain $c_{r}(-\infty<r<\infty)$

$$
\partial_{r+1} \psi_{r} c_{r}+\psi_{r-1} \partial_{r} c_{r}=c_{r}
$$

In particular, if $c_{r}$ is an $r$-cycle, we have

$$
c_{r}=\partial_{r+1}\left(\psi_{r} c_{r}\right)
$$

whence $H^{r}(K)=0$, as was to be proved.

Theorem 3.2. Let $U$ be a normal subgroup of $G$ contained in $H$. Let $A_{U}$ be the submodule of $A$ consisting of elements left fixed by $U$. Then the cohomology groups $H^{r}([G: H], A)$ are isomorphic to the groups $H^{r}\left([G / U: H / U], A_{O}\right),(-\infty<r<\infty)$.

Proof. Let $\eta$ denote the canonical projection map of $G$ onto $G / U$. We choose a fixed set of coset representatives for $G$ modulo $U$; then if $y \in G / U, \eta^{-1}(y)$ shall denote the corresponding coset representative of $y$ in $G$.

Now consider the mappings $f \rightarrow \alpha^{r} f, g \rightarrow \beta^{r} g\left(f \in C^{r}([G: H], A), g \in C^{r}\left([G / U: H / U], A_{O}\right)\right)$ defined by

$$
\begin{aligned}
& \alpha^{r} f\left(\ldots, y_{v}(H / U), \ldots\right)=f\left(\ldots, \eta^{-1}\left(y_{v}\right) H, \ldots\right), \\
& \beta^{r} g\left(\ldots, x_{v} H, \ldots\right) \quad=g\left(\ldots, \eta\left(x_{v}\right)(H / U), \ldots\right) .
\end{aligned}
$$

Then it is easily verified that $\alpha^{r}$ and $\beta^{r}$ set up an isomorphism between $C^{r}([G: H], A)$ and $C^{r}\left([G / U: H / U], A_{U}\right)$. Further, it is clear that $\alpha^{r}$ and $\beta^{r}$ preserve the action of the coboundary operators, and hence induce an isomorphism between the cohomology groups,

$$
H^{r}([G: H], A) \approx H^{r}\left([G / U: H / U], A_{U}\right) .
$$

Corollary. If $H$ is a normal subgroup of $G$, then the cohomology groups $H^{r}([G: H], A)$ are isomorphic to the groups $H^{r}\left(G / H, A_{H}\right),(-\infty<r<\infty)$.

4. Examples. In this section we describe the cohomology groups of the coset space complex $K([G: H])$ in the dimensions $-1,0,1$.

First we remark that a 0 - or $(-1)$-cochain $f$, being a $\bar{G}$-homomorphism, is completely 
determined by its action on the coset consisting of $H$ itself ; for if $f(H)=a$, then $f(x H)=x a$ for all $x \in G$. Clearly also, $a$ lies in the subgroup $A_{H}$ of $A$ consisting of elements left fixed by $H$. Conversely, every element $a$ in $A_{H}$ defines a 0 - and a $(-1)$-cochain, $f_{a}$, given by $f_{a}(x H)=x a$. Consequently, we have

Lemma 4.1. $C^{-1}([G: H], A)$ and $C^{0}([G: H], A)$ are isomorphic to $A_{H}$.

Now let $f=f_{a}$ be a $(-1)$-cochain; $f_{a}$ is a $(-1)$-cocycle if and only if $\delta f_{a}=0$, i.e., if and only if

$$
\delta f_{a}(H)=\Sigma_{v} f_{a}\left(\bar{x}_{\nu} H\right)=\Sigma_{\nu} \bar{x}_{\nu} a=S(a)=0,
$$

where $S(a)$ denotes the trace of $a$ from $A_{H}$ to $A_{G}$ (the submodule consisting of elements left fixed by $G)$. Hence we have

Lemma 4.2. $Z^{-1}([G: H], A)$ is isomorphic to the subgroup of $A_{H}$ consisting of elements with trace zero.

We must now describe the $(-1)$-coboundaries. To this end we decompose $G$ as a union of double cosets modulo $H$ and $H$ :

$$
G=\mathrm{U}_{\nu} H y_{v} H,
$$

with fixed doublo coset representatives $y_{\nu}$. Now $h y_{\nu} H=h^{\prime} y_{\nu} H$ if and only if $h \epsilon h^{\prime}\left(H \cap y_{\nu} H y_{\nu}{ }^{-1}\right)$; thus the single cosets contained in the double coset $H y_{v} H$ are in $(1,1)$ correspondence with the cosets of $H$ modulo $H_{v}=H \cap y_{v} H y_{v}{ }^{-1}$. Hence, if we write

$$
H=\mathrm{U}_{\mu} \bar{h}_{\nu \mu} H_{\nu}
$$

with fixed coset representatives $\bar{h}_{\nu \mu}$, we have

$$
H y_{\nu} H=\mathrm{U}_{\mu} \bar{h}_{\nu \mu} y_{v} H
$$

Further, let $y_{v^{\prime}}$ be the fixed representative of the double coset containing $y_{v}{ }^{-1}$. Then $y_{v}{ }^{-1} H=h^{\prime} y_{v^{\prime}} H$, where $h^{\prime}$ is a coset representative for $H$ modulo $H_{v^{\prime}}$.

Now $f_{a}$ is a $(-1)$-coboundary if and only if there exists a $(-2)$-cochain $g$ such that $f_{a}=\delta g$, i.e., such that

$$
a=f_{a}(H)=\delta g(H)=\Sigma_{v} g\left(\bar{x}_{\nu} H, H\right)-\Sigma_{\nu} g\left(H, \bar{x}_{v} H\right) .
$$

We set $g\left(y_{v} H, H\right)=a_{\nu}$; then $a_{\nu}$ lies in the subgroup $A_{v}$ of $A$ consisting of elements left fixed by $H_{\nu}$. It is easily verified that

$$
\begin{aligned}
& g\left(\bar{h}_{\nu \mu} y_{v} H, H\right)=\bar{h}_{\nu \mu} a_{\nu,} \\
& g\left(H, \bar{h}_{\nu \mu} y_{\nu} H\right)=\left(\bar{h}_{\nu \mu} y_{\nu} h^{\prime}\right) a_{v^{\prime}} .
\end{aligned}
$$

Thus $f_{a}$ is a $(-1)$-coboundary if and only if

$$
\begin{aligned}
a & =\Sigma_{\nu} \Sigma_{\mu}\left(\bar{h}_{\nu \mu} a_{\nu}-\left(\bar{h}_{\nu \mu} y_{\nu} h^{\prime}\right) a_{\nu}\right) \\
& =\Sigma_{\nu} S_{\nu}\left(a_{\nu}-y_{\nu} h^{\prime} a_{\nu^{\prime}}\right),
\end{aligned}
$$

where $S_{v}$ denotes the trace from $A_{\nu}$ to $A_{H}$. Hence,we have

Theorem 4.3. $H^{-1}([G: H], A)$ is isomorphic to the factor group (elements of $A_{\boldsymbol{H}}$ with trace zero) modulo (elements of $A_{B}$ which can be put in the form (*)).

The meaning of this result becomes clearer if we examine the special case obtained by taking $H=1$. Here the double cosets consist simply of single elements of $G$; the $(-1)$ cocycles are determined by the elements of $A$ with zero trace; the (-1)-coboundaries are determined by the elements which can be put in the form

$$
\Sigma_{x \in G}\left(a_{x}-x a_{x}-1\right)=\Sigma_{x \in G}(1-x) a_{x-1} \text {. }
$$


Let now $K \mid k$ be a normal field extension with Galois group $G$, and let $K^{*}$ be the multiplicative group of $K$. Then we have :

Corollary. $H^{-1}\left(G, K^{*}\right)$ is isomorphic to the factor group of (elements with norm 1) modulo (symbolic $(1-x)$-th powers).

Tate has shown, (2), that if $G$ is cyclic, then $H^{-1}\left(G, K^{*}\right)=0$. Hence Theorem 4.3 includes Hilbert's Theorem 90 as a very special case.

We turn now to the 0 -dimensional groups. A 0 -cochain $f=f_{a}$ is a 0 -cocycle if and only if $\delta f_{a}=0$, i.e., if and only if

$$
\delta f_{a}\left(x_{0} H, x_{1} H\right)=f_{a}\left(x_{1} H\right)-f_{a}\left(x_{0} H\right)=x_{1} a-x_{0} a=0,
$$

for all $x_{0}, x_{1} \in G$, i.e., if and only if $a$ lies in $A_{G}$. Thus we have

Lemma 4.4. $Z^{0}([G: H], A)$ is isomorphic to the submodule $A_{G}$ of $A$ consisting of elements left fixed by $G$.

Next, $f_{a}$ is a 0 -coboundary if and only if there exists a $(-1)$-cochain $g=g_{b}\left(b \in A_{H}\right)$ such that $f_{a}=\delta g_{b}$, i:e., such that

$$
a=f_{a}(H)=\delta g_{b}(H)=\Sigma_{\nu} g_{b}\left(\bar{x}_{v} H\right)=\Sigma_{\nu} \bar{x}_{\nu} b=S(b) .
$$

Hence $f_{a}$ is a 0 -coboundary if and only if $a$ is the trace of an element $b$ in $A_{H}$. Thus we have

Theorem 4.5. $H^{0}([G: H], A)$ is isomorphic to the factor group (elements left fixed by $G$ ) modulo (traces of elements left fixed by $H$ ).

Finally, we shall describe the 1-dimensional cohomology group in the case where $G$ operates simply on $A$, i.e., $x a=a$ for all $x \in G, a \in A$. Let $\operatorname{Hom}(G, A)$ denote the group of homomorphisms of $G$ into $A$. Then we shall prove

Theorem 4.6. $H^{1}([G: H], A)$ is isomorphic to the subgroup of $\operatorname{Hom}(G, A)$ consisting of homomorphisms which vanish on $H$.

Proof. First we remark that $B^{1}([G: H], A)=0$. For if $f=\delta g$ is a 1 -coboundary, we have

$$
f\left(x_{0} H, x_{1} H\right)=g\left(x_{1} H\right)-g\left(x_{0} H\right)=x_{1} g(H)-x_{0} g(H)=0 .
$$

Hence $H^{1}([G: H], A)=Z^{1}([G: H], A)$.

Let $\chi$ be an element of $\operatorname{Hom}(G, A)$ which vanishes on $H$. We define a 1-cochain $f_{\chi}$ by writing

$$
f_{\chi}\left(x_{0} H, x_{1} H\right)=\chi\left(x_{0}^{-1} x_{1}\right)
$$

(this does not depend upon the coset representatives $x_{0}, x_{1}$ ). Clearly $f_{x}$ is a 1-cocycle.

Conversely, let $f$ be a 1-cocycle. We define $\chi_{f}$ by setting

$$
\chi_{f}(x)=f(H, x H) \text {. }
$$

Then $\chi_{f}$ is a homomorphism of $G$ into $A$ which vanishes on $H$. This completes the proof.

5. An Exact Sequence. Consider the exact sequence of left $\bar{G}$-modules

$$
0 \rightarrow A^{\prime} \rightarrow A \rightarrow A^{\prime \prime} \rightarrow 0 ;
$$

this is, of course, just a sophisticated way of saying that $A^{\prime}$ is a submodule of $A$ and that $A^{\prime \prime}$ is the factor module. 
Lemma 5.2. If $U$ is a subgroup of $G$ with the property that $H^{1}\left(U, A^{\prime}\right)=0$, then the sequence

$$
0 \rightarrow A_{U}^{\prime} \rightarrow A_{U} \rightarrow A_{U}^{\prime \prime} \rightarrow 0
$$

is exact (where these are the subgroups left fixed by $U$ ).

Proof. All we have to show is that the mapping of $A_{U}$ into $A_{U}^{\prime \prime}$ is onto. So let $a^{\prime \prime} \in A_{V}^{\prime \prime}$. Let $b$ be any element of $A$ lying in the coset $a^{\prime \prime}$. Then for any elements $u, v \in U$, we have $u\left(b+A^{\prime}\right)=v\left(b+A^{\prime}\right)$, whence $u b-v b$ lies in $A^{\prime}$.

We now define $f \in C^{1}\left(U, A^{\prime}\right)$ by setting $f(u, v)=u b-v b$; clearly $f$ is a 1-cocycle. Since $H^{1}\left(U, A^{\prime}\right)=0$, there exists an element $\beta \in A^{\prime}$ defining a 0 -cochain, $g(u)=u \beta$, such that $f=\delta g$, i.e., such that $u b-v b=v \beta-u \beta$. Then clearly $a=b+\beta \in A_{U}$, and lies in the coset $a^{\prime \prime}$. This completes the proof.

Lemma 5.3. If $H^{1}\left(U, A^{\prime}\right)=0$ for all subgroups $U$ of $H$, then for every cochain

$$
f \in C^{r}\left([G: H], A^{\prime \prime}\right) \text {. }
$$

there exists a cochain $g \in C^{r}([G: H], A)$ such that $j(g(c))=f(c)$ for all $c \in C_{r}([G: H])(j$ denotes the canonical projection of $A$ onto $\left.A^{\prime \prime}\right)$.

Proof. Let $f \in C^{r}\left([G: H], A^{\prime \prime}\right)(r \geqslant 0)$ and let $\sigma^{1}=\left(H, x_{1} H, \ldots, x_{n} H\right)$. Then $\sigma^{1}$ is left fixed by $U=H \cap x_{1} H x_{1}^{-1} \cap \ldots \cap x_{n} H x_{n}^{-1}$, which is a subgroup of $H$. Hence $f\left(\sigma^{1}\right)$ is left fixed by $U$ also, since $f$ is a $\bar{G}$-homomorphism. According to Lemma 5.2 and the hypothesis that $H^{1}\left(U, A^{\prime}\right)=0$, there exists an element $a_{1} \in A_{U}$ such that $j a_{1}=f\left(\sigma^{1}\right)$. We define $g\left(\sigma^{1}\right)=a_{1}$; then for every $x \in G$ we define $g\left(x \sigma^{1}\right)=x a_{1}$.

If the collection $\left\{x \sigma^{1}\right\}$ does not exhaust the generators of $C_{r}$, let $\sigma^{2}$ be another generator, not in this collection, with first "vertex" $H$. We proceed in the same way with $\sigma^{2}$ and continue until $g$ has been defined on all the generators of $C_{r}$.

Since $C_{-r}([G: H])$ is $\bar{C}_{r}$-isomorphic to $C_{r-1}([G: H])$, the required result follows at once for the negative-dimensional groups also. This completes the proof.

The injection map, $i: A^{\prime} \rightarrow A$, and the projection map, $j: A \rightarrow A^{\prime \prime}$, induce cohomology group homomorphisms

$$
\begin{aligned}
& i_{r}^{*}: H^{r}\left([G: H], A^{\prime}\right) \rightarrow H^{r}([G: H], A), \\
& j_{*}^{r}: H^{r}([G: H], A) \rightarrow H^{r}\left([G: H] A^{\prime \prime}\right) .
\end{aligned}
$$

It is well known that, by virtue of Lemma 5.3, we can construct a third homomorphism

$$
d_{*}^{r}: H^{r}\left([G: H], A^{\prime \prime}\right) \rightarrow H^{r+1}\left([G: H], A^{\prime}\right) .
$$

Namely, if $f \in Z^{r}\left([G: H], A^{\prime \prime}\right)$, and $g$ is a cochain in $C^{r}([G: H], A)$.such that $j(g(c))=f(c)$, then $\delta g$ lies in $Z^{r+1}\left([G: H], A^{\prime}\right)$, and the correspondence

$$
f \rightarrow \delta g
$$

induces the homomorphism $d_{*}^{*}$ of the cohomology groups. It is then easy to establish

Theorem 5.4. If the exact sequence (5.1) is such that $H^{1}\left(U, A^{\prime}\right)=0$ for all subgroups $U$ of $H$, then the sequence

$$
\ldots \rightarrow H^{r}\left([G: H], A^{\prime}\right) \rightarrow H^{r}([G: H], A) \rightarrow H^{r}\left([G: H], A^{\prime \prime}\right) \rightarrow H^{r+1}\left([G: H], A^{\prime}\right) \rightarrow \ldots
$$

may be defined and is exact, $-\infty<r<\infty$.

Corollary. For any exact sequence (5.1), the sequence

$$
\ldots \rightarrow H^{r}\left(G, A^{\prime}\right) \rightarrow H^{r}(G, A) \rightarrow H^{r}\left(G, A^{\prime \prime}\right) \rightarrow H^{r+1}\left(G, A^{\prime}\right) \rightarrow \ldots
$$

may be defined and is exact, $-\infty<r<\infty$. 
6. Some Special Coefficient Modules. We recall that in the proof of Theorem 3.1 we introduced mappings $\psi_{r}: C_{r}([G: H]) \rightarrow C_{r+1}([G: H])$ with the property that $c=\partial_{r+1} \psi_{r} c+\psi_{r-1} \partial_{r} c$ for all $c \epsilon C_{r}$. We remark that these mappings are $\bar{H}$-homomorphisms.

Lemma 6.1. Let $A$ and $B$ be left $\bar{G}$-modules, $\phi$ a $\bar{G}$-homomorphism of $A$ into $B$ such that for every $a \epsilon A, \phi(a)=\Sigma_{\nu} \bar{x}_{\nu} \theta\left(\bar{x}_{\nu}^{-1} a\right)$, where $\theta$ is an $\bar{H}$-homomorphism of $A$ into $B$. Then the induced cohomology group homomorphisms $\phi_{*}^{*}: H^{r}([G: H], A) \rightarrow H^{r}([G: H], B)$ are zero, $-\infty<r<\infty$.

Proof. Let $\phi^{r}$ be the cocycle group homomorphism induced by $\phi$. If $f \in Z^{r}([G: H], A)$, then $\phi^{r} f \in Z^{r}([G: F], B)$ is given by $\left(\phi^{r} f\right)(c)=\phi(f(c)), c \in C^{r}([G: H])$. By hypothesis, this may be written

$$
\left(\phi^{r} f\right)(c)=\Sigma_{\nu} \bar{x}_{\nu} \theta\left(\bar{x}_{\nu}^{-1} f(c)\right)=\Sigma_{\nu} \bar{x}_{\nu} \theta\left(f\left(\bar{x}_{\nu}^{-1} c\right)\right) .
$$

Next, since $\bar{x}_{\nu}^{-1} c=\partial_{r+1} \psi_{r}\left(\bar{x}_{\nu}^{-1} c\right)+\psi_{r-1} \partial_{r}\left(\bar{x}_{\nu}^{-1} c\right)$, and $f$ is a cocycle, we obtain

$$
\left(\phi^{r} f\right)(c)=\Sigma_{v} \bar{x}_{v} \theta\left(f\left(\psi_{r-1} \partial_{r} \bar{x}_{\nu}^{-1} c\right)\right)=\Sigma_{v}\left(\bar{x}_{\nu} \theta f \psi_{r-1} \bar{x}_{\nu}^{-1}\right)\left(\partial_{r} c\right)=\delta g(c)
$$

where $g=\Sigma_{\nu} \bar{x}_{\nu} \theta f \psi_{r-1} \bar{x}_{\nu}^{-1}$ is a $\bar{G}$-homomorphism (and hence an $(r-1)$-cochain) since $\theta$ and $\psi_{r-1}$ are $\bar{H}$-homomorphisms. Hence $\phi_{*}^{r}=0$.

A left $\bar{G}$-module $A$ is said to be semi-regular if it contains an $\bar{H}$-module $B$ such that $A=\Sigma_{\nu} \bar{x}_{v} B$, i.e., if every element $a \in A$ can be written uniquely in the form $a=\Sigma_{\nu} \bar{x}_{\nu} b_{v}$, with $b_{\nu} \in B$. The mapping $e^{\prime}: A \rightarrow A$ given by

$$
e^{\prime}\left(\sum_{\nu} \bar{x}_{\nu} b_{v}\right)=b_{1}
$$

(where $\bar{x}_{1}=1$ is the coset representative of $H$ itself) is clearly an $\bar{H}$-homomorphism. Further, if $e$ is the identity map of $A$ onto itself, we have

$$
e(a)=a=\Sigma_{\nu} \bar{x}_{\nu} e^{\prime}\left(\bar{x}_{v}^{-1} a\right) .
$$

Thus the identity map of a semi-regular $\bar{G}$-module satisfies the condition of Lemma 6.1 . Since the identity map of $A$ onto $A$ induces the identity map of the cohomology groups, we have

Theorem 6.2. If $A$ is a semi-regular $\bar{G}$-module, then $H^{r}([G: H], A)=0,-\infty<r<\infty$.

A left $\bar{G}$-module $A$ is said to be regular if it contains a subgroup $B$ such that $A=\sum_{x \in G} x B$. Clearly such a module can be written $A=\Sigma_{\nu} \bar{x}_{\nu} B^{\prime}$, where $B^{\prime}=\Sigma_{h \in B} h B$. Since $B^{\prime}$ is an $\bar{H}$-module it follows that every regular $\bar{G}$-module is also semi-regular, and we have

Theorem 6.3. If $A$ is a regular $\bar{G}$-module, then $H^{r}([G: H], A)=0,-\infty<r<\infty$. In particular, $H^{r}([G: H], \bar{G})=0,-\infty<r<\infty$.

Let now $A$ be a left $\bar{G}$-module satisfying the conditions

(6.4) $\quad G$ acts simply on $A$, i.e., $x a=a$ for all $x \in G, a \in A$;

(6.5) the mapping $a \rightarrow m a$ is an automorphism of $A$, where $m$ is the index of $H$ in $G$.

Under these conditions, the mapping $e^{\prime}: A \rightarrow A$ given by $e^{\prime}(a)=\frac{1}{m} a$ is an $\bar{H}$-homomorphism ; if $e$ is the identity map of $A$ onto itself, we have

$$
e(a)=a=\Sigma_{\nu} \bar{x}_{\nu} e^{\prime}\left(\bar{x}_{\nu}^{-1} a\right) .
$$

Hence, appealing to Lemma 6.1 again, we have 
7'heorem 6.6. If $A$ is a left $\bar{G}$-module satisfying (6.4) and (6.5), then $H^{r}([G: H], A)=0$. In particular, $H^{r}([G: H], R)=0$ where $R$ denotes the additive group of real numbers with simple action by $G$.

Let $Z$ denote the group of integers, with simple action by $G$. Then we have, as immediate consequences of Theorems 4.3, 4.5, 4.6,

Theorem 6.7. $H^{-1}([G: H], Z)=H^{1}([G: H], Z)=0 ; H^{0}([G: H], Z)$ is cyclic of order $m$.

Finally, let $T=R / Z$ be the group of real numbers modulo integers, also with simple action by $G$. Then the exact sequence

$$
0 \rightarrow Z \rightarrow R \rightarrow T \rightarrow 0
$$

satisfies the condition of Theorem 5.4. Hence we have an exact sequence

$$
\ldots \rightarrow H^{r}([G: H], R) \rightarrow H^{r}([G: H], T) \rightarrow H^{r+1}([G: H], Z) \rightarrow H^{r+1}([G: H], R) \rightarrow \ldots
$$

By virtue of Theorem 6.6 , this yields

Theorem 6.8. $H^{r}([G: H], T)$ is isomorphic to $H^{r+1}([G: H], Z)$.

Now Hom $(G, T)$ is the character group, Char $G$, of $G$. According to Theorem 4.6, $H^{1}([G: H], T)$ is isomorphic to the subgroup of Char $G$ consisting of characters which vanish on $H$. This, as is well known, is isomorphic to the character group of $G / G^{\prime} H$, where $G^{\prime}$ is the commutator subgroup of $G$. Hence we have

Corollary. $H^{2}([G: H], Z)$ and $H^{1}([G: H], T)$ are isomorphic to Char $\left(G / G^{\prime} H\right)$.

7. Link between the Cohomology Groups of $G, H$ and $[G: H]$. The inflation (lifting) homomorphisms $\lambda^{r}: C^{r}([G: H], A) \rightarrow C^{r}(G, A)$ are defined by

$$
\left(\lambda^{r} f\right)\left(x_{0}, \ldots, x_{r}\right)=f\left(x_{0} H, \ldots, x_{r} H\right), f \in C^{r}([G: H], A) .
$$

Clearly $\delta_{r} \lambda^{r}=\lambda^{r+1} \delta_{r}$; hence the homomorphisms $\lambda^{r}$ induce corresponding homomorphisms $\lambda_{*}^{r}$ of the cohomology groups. The restriction homomorphisms, $\rho^{r}: C^{r}(G, A) \rightarrow C^{r}(H, A)$ are defined by

$$
\left(\rho^{r} g\right)\left(h_{0}, \ldots, h_{r}\right)=g\left(h_{0}, \ldots, h_{r}\right), \quad g \in C^{r}(G, A) ;
$$

these also induce cohomology group homomorphisms $\rho_{*}^{r}$. We are thus led to consider the sequence

$$
0 \rightarrow H^{n}([G: H], A) \rightarrow H^{n}(G, A) \rightarrow H^{n}(H, A) .
$$

Our aim is to show that under certain conditions this sequence is exact. To this end we prove first

Lemma 7.2. Every left $\bar{G}$-module $A$ can be embedded isomorphically in a regular $\bar{G}$-module.

Proof. Let $A$ be the additive abelian group formed by all arbitrary maps $f: G \rightarrow A$. We define an action of $G$ on $A$ by setting $(x f)(y)=f(y x),(x, y \in G)$. Then $\bar{A}$ is a regular $\bar{G}$-module; for the maps $f$ such that $f(x)=0$ for $x \neq 1$ form a subgroup $B$, and $\bar{A}=\Sigma_{x \in} a x B$. $A$ is then mapped isomorphically into $\bar{A}$ by the correspondence $a \rightarrow f_{a}$ where $f_{a}(x)=x a$.

Theorem 7.3. Let $H^{v}(U, A)=0$ for all subgroups $U$ of $H$ and for $v=1,2, \ldots, n-1$. Then the sequence (7.1) is exact.

Proof. We proceed by induction on $n$.

First, let $n=1$. Let $f \in Z^{1}([G: H], A)$ be such that $\lambda f=\delta g$, where $g \in C^{0}(G, A)$. We must show that $f=\delta g^{\prime}$, where $g^{\prime} \in C^{0}([G: H], A)$. We remark that the values of $g$ depend only on the coset of its argument modulo $H$; for

$$
g(x)-g(x h)=(\lambda f)(x h, x)=f(x H, x H)=0,
$$


since $\delta f(x H, x H, x H)=0$. Hence we may define $g^{\prime} \in C^{0}([G: H], A)$ by setting $g^{\prime}(x H)=g(x)$; clearly $f=\delta g^{\prime}$, so we have proved exactness at the first stage.

Next let $f \in Z^{1}(G, A)$ be such that $\rho f=\delta g$, where $g \in C^{0}(H, A)$. We must show that $f$ differs by at most a coboundary from a cocycle of the form $\lambda f^{\prime}$, where $f^{\prime} \in C^{1}([G: H], A)$. With this aim, we extend $g$ to a 0 -cochain $g^{\prime} \in C^{0}(G, A)$ by setting $g^{\prime}(x)=x g(1)$. Then let $e=f-\delta g^{\prime}$; we claim that $e$ is a cocycle whose values depend only on the cosets of its arguments modulo $H$. This follows from the formulae

$$
\begin{aligned}
& 0=\delta e\left(x_{0} h_{1}, x_{0} h_{2}, x_{1}\right)=e\left(x_{0} h_{2}, x_{1}\right)-e\left(x_{0} h_{1}, x_{1}\right)+e\left(x_{0} h_{1}, x_{0} h_{2}\right), \\
& 0=\delta e\left(x_{0}, x_{1} h_{1}, x_{1} h_{2}\right)=e\left(x_{1} h_{1}, x_{1} h_{2}\right)-e\left(x_{0}, x_{1} h_{2}\right)+e\left(x_{0}, x_{1} h_{1}\right),
\end{aligned}
$$

when we notice that $e\left(x_{0} h_{1}, x_{0} h_{2}\right)=x_{0} e\left(h_{1}, h_{2}\right)=0$, and similarly $e\left(x_{1} h_{1}, x_{1} h_{2}\right)=0$. Then it is clear that $e=\lambda f^{\prime}$, where we define $f^{\prime}$ by setting $f^{\prime}\left(x_{0} H, x_{1} H\right)=e\left(x_{0}, x_{1}\right)$. This completes the proof of the exactness at the second stage, and hence establishes a basis for the induction.

Let us now suppose that we have established the theorem for $n=k \geqslant 1$, and, further, that the conditions of the theorem are satisfied for $n=k+1$. We embed the module $A$ in a regular $\bar{G}$-module $A$; let $A^{*}$ be the factor module. Then the exact sequence

$$
0 \rightarrow A \rightarrow A \rightarrow A^{*} \rightarrow 0
$$

satisfies the condition of Theorem 5.4. Hence we have exact sequences

$$
\ldots \rightarrow H^{\nu}(\bar{A}) \rightarrow H^{\nu}\left(A^{*}\right) \rightarrow H^{v+1}(A) \rightarrow H^{v+1}(\bar{A}) \rightarrow \ldots
$$

for the cohomology groups of $G, H,[G, H]$, and all subgroups $U$ of $H$. Since $A$ is regular, all the groups $H^{\nu}(\bar{A})$ are zero, and hence we obtain isomorphisms

$$
H^{v}\left(A^{*}\right) \approx H^{v+1}(A)
$$

Thus the factor module $A^{*}$ satisfies the conditions of the Theorem with $n=k$. It follows that in the diagram

$$
\begin{aligned}
& 0 \rightarrow H^{k}\left([G: H], A^{*}\right) \rightarrow H^{k}\left(G, A^{*}\right) \rightarrow H^{k}\left(H, A^{*}\right) \\
& \underset{0 \rightarrow H^{k+1}([G: H], A)}{\downarrow} \rightarrow H^{k+1}(G, A) \rightarrow H^{k+1}(H, A)
\end{aligned}
$$

the upper sequence is exact. Since the inflation and restriction maps clearly commute with the isomorphisms (7.4) it follows that the lower sequence is exact. This establishes the induction and hence completes the proof of the theorem.

Corollary. Under the conditions of Theorem $7.3, H^{n}([G: H], A)$ is isomorphic to the subgroup of $H^{n}(G, A)$ consisting of cohomology classes which split on restriction to $H$.

8. Cohomology Groups for Arbitrary Field Extensions. Let $E \mid k$ be an arbitrary field extension of finite degree. Let $K$ be a normal extension of $k$ containing $E$; let $G$ be the Galois group of $K \mid k, H$ the subgroup of $G$ which leaves $E$ fixed. Let $A_{K}$ denote either the multiplicative group, $K^{*}$, of non-zero elements of $K$, or (in the case where $K$ is a field to which global class field theory applies) the group of idèles, $J_{K}$, or the group of idele classes, $C_{K}$, of $K$. These groups are all $\bar{G}$-modules (actually they are written multiplicatively, but this causes us no alarm). Let $A_{E}$ and $A_{k}$ denote the corresponding groups for $E$ and $k$. Then, as is well known, in each of the cases we are considering, $A_{E}$ and $A_{k}$ may be identified with the subgroups of $A_{K}$ left fixed by $H$ and $G$ respectively. 
Using the auxiliary normal extension $K$, we define cohomology groups for the "layers" $A_{E} \mid A_{k}$ by setting

$$
H_{K}^{r}\left(A_{E} \mid A_{k}\right)=H^{r}\left([G: H], A_{K}\right) .
$$

Concerning these, we prove

Theorem 8.1. The cohomology groups $H_{K}^{r}\left(A_{E} \mid A_{k}\right)$ do not depend on the normal extension $K$.

Proof. Let $K$ and $K^{\prime}$ be normal extensions of $k$ containing $E$. First we consider the case in which $K$ contains $K^{\prime}$. Let $U$ be the normal subgroup of $G$ which leaves $K^{\prime}$ fixed. Then, according to Theorem 3.2 ,

$$
H_{K}^{r}\left(A_{E} \mid A_{k}\right)=H^{r}\left([G: H], A_{K}\right) \approx H^{r}\left([G / U: H / U], A_{K^{\prime}}\right)=H_{K^{\prime}}^{r}\left(A_{E} \mid A_{k}\right) .
$$

Finally, if $K$ and $K^{\prime}$ are any two normal extensions of $k$ containing $E$, then so is $K \cap K^{\prime}$, and we have

$$
H_{K}^{r}\left(A_{E} \mid A_{k}\right) \approx H_{K^{\prime} K^{\prime}}^{r}\left(A_{E} \mid A_{k}\right) \approx H_{K^{\prime}}^{r}\left(A_{E} \mid A_{k}\right) .
$$

This completes the proof.

Henceforth we may omit all reference to the auxiliary normal extension, and write the groups simply as $H^{r}\left(A_{E} \mid A_{k}\right)$.

Corollary. If $E$ is itself a normal extension, with Galois group $G$, then $H^{r}\left(A_{E} \mid A_{k}\right)$ as defined above is isomorphic to $H^{r}\left(G, A_{E}\right)$.

9. The Cohomology Groups of Class Field Theory. As in the preceding section, let $E \mid k$ be an arbitrary extension of finite degree ; let $K$ be a normal extension of $k$ containing $E$; let $G$ and $H$ be as before.

It is an immediate consequence of Theorem 7.3 that if $H^{1}\left(U, A_{K}\right)=0$ for all subgroups $U$ of $G$, then $H^{1}\left([G: H], A_{K}\right)=H^{1}\left(A_{E} \mid A_{k}\right)=0$ also. This condition is satisfied in the cases under consideration, $A_{K}=K^{*}, J_{K}, C_{K}$ (2), (5). Thus we have

Theorem 9.1. For all field extensions $E \mid k$, normal or not, $H^{1}\left(E^{*} \mid k^{*}\right)=0, H^{1}\left(J_{E} \mid J_{k}\right)=0$, $H^{1}\left(C_{E} \mid C_{k}\right)=0$.

Next, from the Corollary to Theorem 7.3 it follows that if $H^{1}\left(U, A_{K}\right)=0$ for all subgroups $U$ of $H$, then $H^{2}\left([G: H], A_{K}\right)=H^{2}\left(A_{E} \mid A_{k}\right)$ is isomorphic to the subgroup of $H^{2}\left(G, A_{K}\right)$ consisting of cohomology classes which split on restriction to $H$. According to (3), this yields the following description of the 2-dimensional cohomology group in the case $A_{K}=K^{*}$ :

Theorem 9.2. $H^{2}\left(E^{*} \mid k^{*}\right)$ is isomorphic to the group of central simple algebra classes over $k$ which have $E$ as splitting field.

Let now $k$ be a field for which class field theory holds. For every finite extension $E$ of $k$, let $A_{E}$ denote either the multiplicative group of non-zero elements of $E$ (local case) or the group of idele classes of $E$ (global case). Then it is well known, (2), (5), that for normal extensions $K \mid k, H^{2}\left(A_{K} \mid A_{k}\right)$ is cyclic of degree equal to the degree of $K \mid k$, and is generated by a canonical cohomology class $\gamma(K \mid k)=\gamma$ which has (inter alia) the property that $\gamma^{i}$ splits on a subfield $E$ of $K$ if and only if $i$ is divisible by the degree of $K \mid E$. Thus we have

Theorem 9.3. For all field extensions $E \mid k$, normal or not, $H^{2}\left(A_{E} \mid A_{k}\right)$ is cyclic of order equal to the degree of $E \mid k$; it has a canonical generator, $\gamma^{d}$, where $d=\operatorname{deg}(K \mid E)$ and $\gamma$ is the canonical generator for $H^{2}\left(A_{K} \mid A_{k}\right)$. 
We now give a non-arithmetic model for the cohomology groups $H^{2}\left(A_{E} \mid A_{k}\right)$ for an arbitrary extension. As in (6), let $\bar{A}$ denote the splitting group over $A_{K}$ for $\gamma(K \mid k)$. Then we have $H^{r}(U, A)=0$ for all subgroups $U$ of $G, r>0$. Hence from Theorem 7.3 it follows at once that $H^{r}([G: H], \bar{A})=0, r>0$. We now use the exact sequence

$$
0 \rightarrow A_{K} \rightarrow \bar{A} \rightarrow I \rightarrow 0,
$$

where $I$ denotes the submodule of the group ring $\bar{G}$ consisting of elements $\Sigma a_{x} x$ such that $\Sigma a_{x}=0$. This sequence satisfies the condition of Theorem 5.4; hence we have an exact sequence

$$
\ldots \rightarrow H^{r}([G: H], A) \rightarrow H^{r}([G: H], I) \rightarrow H^{r+1}\left([G: H], A_{K}\right) \rightarrow H^{r+1}([G: H], A) \rightarrow \ldots
$$

Since the extreme groups are zero (see the remark above), we obtain ·

Theorem 9.4. For $r>0, H^{r+1}\left(A_{E} \mid A_{k}\right)$ is isomorphic to $H^{r}([G: H], I)$.

This is certainly less satisfactory than Tate's result, but we cannot use (as he does) the exact sequence

$$
0 \rightarrow I \rightarrow \bar{G} \rightarrow Z \rightarrow 0,
$$

since it does not satisfy the condition of Theorem 5.4. To overcome this difficulty, Dr. Y. Kawada has suggested to the author that we use certain modified cohomology groups,

$$
\underline{H}^{r}([G: H], Z)=\left[Z^{r}([G: H], Z) \cap j Z^{r}([G: H], \bar{G})\right] / j B^{r}([G: H], \bar{G}) .
$$

Clearly we can then obtain an exact sequence

$$
\ldots \rightarrow H^{r}([\bar{G}: H], G) \rightarrow \underline{H}^{r}([G: H], Z) \rightarrow H^{r+1}([G: H], I) \rightarrow H^{r+1}([G: H], \bar{G}) \rightarrow \ldots
$$

Since the cohomology groups with coefficients in $\bar{G}$ are zero (Theorem 6.3), this exact sequence yields isomorphisms,

$$
\underline{H}^{r}([G: H], Z) \approx H^{r+1}([G: H], I), \quad-\infty<r<\infty .
$$

Combining these isomorphisms with Theorem 9.4, we obtain

Theorem 9.5. For $r>0, \bar{H}^{r+1}\left(A_{\boldsymbol{E}} \mid A_{k}\right)$ is isomorphic to the modified cohomology group $\underline{H}^{r-1}([G: H], Z)$.

Since, in particular, $\underline{H}^{1}([G: H], Z)=0$, we see that $H^{3}\left(A_{E} \mid A_{k}\right)=0$. But in general the modified cohomology groups appear to be very complicated.

\section{REFERENCES}

(1) E. Artin, Algebraic Numbers and Algebraic Functions (New York, 1951).

(2) E. Artin and J. T. Tate, Class Field Theory, Princeton seminar notes, 1951-2, (to appear).

(3) R. Brauer, "On Splitting Fields of Simple Algebras", Annals of Math., vol. 48 (1947), pp. 79-90.

(4) S. Eilenberg, "Homology of Spaces with Operators, I ", Trans. Amer. Math. Soc., vol. 61 (1947), pp. 378-417.

(5) G. Hochschild and T. Nakayama, "Cohomology in Class Field Theory ", Annals of Math., vol. 55 (1952), pp. 348-66.

(6) J. T. Tate, "The Higher Dimension Cohomology Groups of Class Field Theory ", Annals of Math., vol. 56 (1952), pp. 294-7.

\section{The Quenn's University of Belfast}

